\title{
Co-treatment of THP-1 cells with naringenin and curcumin induces cell cycle arrest and apoptosis via numerous pathways
}

\author{
DUNYUN SHI ${ }^{1}$, YUN XU $^{2}$, XIN DU $^{1}$, XUHONG CHEN $^{2}$, XIAOLI ZHANG ${ }^{2}$, \\ JIN LOU ${ }^{1}$, MING LI ${ }^{1}$ and JIACAI ZHUO ${ }^{1}$ \\ ${ }^{1}$ Institute of Hematology; ${ }^{2}$ Central Laboratory, Shenzhen Second People's Hospital, \\ Shenzhen, Guangdong 518035, P.R. China
}

Received December 22,2014; Accepted September 16, 2015

DOI: $10.3892 / \mathrm{mmr} .2015 .4480$

\begin{abstract}
Acute myeloid leukemia (AML) is a hematological malignancy with a low survival rate. Curcumin, which is a multi-targeted anticancer agent, has been shown to exert anti-oxidant, anti-inflammatory, anti-mutagenic and anti-carcinogenic activities. Naringenin is extracted from citrus fruits and exerts anti-mutagenic and anti-carcinogenic activities in various types of cancer cells. However, the effects of curcumin and naringenin in combination in AML cells have yet to be studied. The present study aimed to investigate the combination effects of curcumin and naringenin on the viability, cell cycle distribution and apoptosis rate of THP-1 cells using cell viability assays, flow cytometry, and western blotting. Naringenin enhanced curcumin-induced apoptosis and cell viability inhibition. In addition, curcumin and naringenin induced cell cycle arrest at $\mathrm{S}$ phase and $\mathrm{G} 2 / \mathrm{M}$ phase. Numerous pathways, including p53, c-Jun N-terminal kinases (JNK), Akt and extracellular signal-regulated kinases (ERK)1/2 pathways were markedly altered following treatment of THP-1 cells with curcumin and naringenin. These results indicated that naringenin may enhance curcumin-induced apoptosis through inhibiting the Akt and ERK pathways, and promoting the JNK and p53 pathways.
\end{abstract}

\section{Introduction}

Acute myeloid leukemia (AML) is a hematological malignancy characterized by a clonal disorder of myeloid hematopoietic stem and progenitor cells (1). In AML, the proliferation of malignant hematopoietic cells is dysregulated and normal hematopoietic functions are lost; therefore, numerous patients succumb to AML within a short period. AML is a common

Correspondence to: Dr Jiacai Zhuo, Institute of Hematology, Shenzhen Second People's Hospital, 3002 Sungang West Road, Shenzhen, Guangdong 518035, P.R. China

E-mail: zhuojiacai@126.com

Key words: naringenin, curcumin, cell cycle arrest, apoptosis, THP-1 cells type of hematological cancer in adults, which is associated with a low survival rate (2). The main therapeutic strategies used to treat AML include intensive chemotherapy and hematopoietic stem cell transplantation. The majority of patients with AML enter remission following aggressive chemotherapy; however, relapse often occurs in a large number of patients. Therefore, novel and more effective drugs or therapeutic strategies are warranted, in order to improve the treatment and prognosis of patients with AML.

Curcumin, which is extracted from the plant Curcuma longa (3), has numerous biological activities and its use in inflammatory and infectious diseases has been extensively investigated in the past few decades (4). It has previously been reported that curcumin exerts anti-oxidant, anti-inflammatory, anti-mutagenic and anti-carcinogenic activities (5). Furthermore, it has been suggested that curcumin inhibits proliferation and induces apoptosis through the hyperproduction of reactive oxygen species (ROS) in solid tumor cells (6), or through an ornithine decarboxylase-dependent pathway in promyelocytic leukemia cells (7). In addition, numerous pathways including the cell survival pathway, caspase activation pathway, protein kinase pathway and death receptor pathway are associated with curcumin-induced modulation of tumor cells (8). The antitumor activity and underlying mechanisms of curcumin have been well investigated in various types of tumor cells, thus suggesting that curcumin may be considered an effective antitumor drug; however, the effects of curcumin on AML cells have yet to be investigated.

Naringenin is a flavonoid that is predominantly extracted from citrus fruits, and exerts anti-mutagenic and anti-carcinogenic activities (9-11). Previous studies have demonstrated that naringenin may inhibit growth and induce apoptosis of hepatocellular and epidermoid carcinoma cells, through ROS generation and cell cycle arrest $(12,13)$. Naringenin and curcumin have exhibited antitumor activity in various types of solid cancer cells $(5,6,9,11)$; however, the antitumor activity of naringenin, and the effects of naringenin in combination with curcumin on AML cells are yet to be elucidated.

The present study aimed to investigate the effects of a combination of naringenin and curcumin on THP-1 monocytic leukemia cells. The results revealed that naringenin enhanced curcumin-induced cell cycle arrest and apoptosis of THP-1 
cells, and inhibition of the Akt and p53 pathways may be involved in these actions.

\section{Materials and methods}

Cells and reagents. THP-1 human myeloid leukemia cells (American Type Culture Collection, Mannassas, VA, USA) were maintained in RPMI-1640 medium (Gibco; Thermo Fisher Scientific, Inc., Waltham, MA, USA) supplemented with $10 \%$ heat-inactivated fetal bovine serum (Zhejiang Tianhang Biotechnology Co., Ltd., Zhejiang, China), glutamine (Gibco; Thermo Fisher Scientific, Inc.), and penicillin/streptomycin (Weihong Biotechnology Co. Ltd, Shanghai, China) at $37^{\circ} \mathrm{C}$ in an atmosphere containing 5\% $\mathrm{CO}_{2}$. Curcumin and naringenin were purchased from Sigma-Aldrich (St. Louis, MO, USA). 3-(4,5-dimethylthiazol-2-yl)-2,5-diphenyltetrazolium bromide (MTT) was obtained from Beyotime Institute of Biotechnology (Shanghai, China).

Antibodies. Rabbit-anti human phosphorylated (p)-c-Jun $\mathrm{N}$-terminal kinases (JNK) monoclonal antibody (mAb; cat. no. 4668), rabbit-anti human JNK polyclonal antibody (pAb; cat. no. 9252), mouse-anti human p-Akt mAb (cat. no. 2920) and rabbit-anti human Akt pAb (cat. no. 9272) were purchased from Cell Signaling Technology, Inc. (Danvers, MA, USA). Mouse-anti human p53 mAb (cat. no. sc-126), mouse-anti human p-extracellular signal-regulated kinases (ERK) $1 / 2$ mAb (cat. no. sc-7383) and rabbit-anti human ERK1/2 pAb (cat. no. sc-94) were purchased from Santa Cruz Biotechnology, Inc. (Dallas, TX, USA). Mouse-anti human $\beta$-actin mAb (cat.no. AA128), rabbit-anti human caspase-3 pAb (cat.no. AC030), and horseradish peroxidase (HRP)-conjugated goat-anti mouse and rabbit immunoglobulin $\mathrm{G}$ were purchased from Beyotime Institute of Biotechnology.

Cell viability assay. The effects of curcumin, naringenin or a combination of the two on cell viability were determined using the MTT cell viability assay. Briefly, $5 \times 10^{4}$ cells were plated in a 96-well plate and incubated with the specified drugs at indicated concentrations $(25,50,100,200$ or $400 \mu \mathrm{m}$ for naringenin, 20 or $40 \mu \mathrm{m}$ for curcumin) for 24 or $48 \mathrm{~h}$. Following incubation, MTT solution $(0.5 \mathrm{mg} / \mathrm{ml})$ was added and the cells were incubated for a further $3 \mathrm{~h}$. A solution (100 $\mu \mathrm{l})$ containing $10 \%$ sodium dodecyl sulfate (SDS) and $0.01 \mathrm{M} \mathrm{HCl}$ was then added to dissolve the crystals. The absorbance was measured using a microplate reader (Bio-Rad Laboratories, Inc., Hercules, CA, USA) at a test wavelength of $570 \mathrm{~nm}$. Cell viability was determined as a percentage of the vehicle control. All cell viability assays were performed in triplicate and repeated in three independent experiments.

Cell cycle analysis. Cells treated with curcumin, naringenin or a combination of the two for $48 \mathrm{~h}$ were collected, washed with phosphate-buffered saline (PBS), fixed in cold 70\% ethanol and incubated at $4^{\circ} \mathrm{C}$ overnight. The fixed cells $\left(5 \times 10^{5}\right)$ were subsequently washed with PBS, and incubated with RNase A $(100 \mu \mathrm{g} / \mathrm{ml}$; Beyotime Institute of Biotechnology) and propidium iodide (PI; $50 \mu \mathrm{g} / \mathrm{ml}$ ) for $30 \mathrm{~min}$ on ice. The DNA content was determined by fluorescence-activated cell sorting using a BD FACSCalibur flow cytometer (BD Biosciences, Franklin
Lakes, NJ, USA), and the cell cycle profile was evaluated using FlowJo v10 software (Tree Star, Inc., Ashland, OR, USA).

Apoptosis assay. Following treatment with curcumin, naringenin or a combination of the two for $48 \mathrm{~h}$, the cells were collected and washed twice with PBS. Apoptosis was detected following Annexin-V and PI staining, which was conducted using the Annexin-V-Fluorescein Isothiocyanate Apoptosis Analysis kit (Beyotime Institute of Biotechnology), according to the manufacturer's protocol. Analysis was performed on a flow cytometer (BD Biosciences).

Western blot analysis. Whole cell lysates were extracted from the cells suspended in radioimmunoprecipitation buffer (Beyotime Institute of Biotechnology) supplemented with $1 \mathrm{mM}$ phenylmethylsulfonyl fluoride (Beyotime Institute of Biotechnology). Cell lysates were separated by electrophoresis on polyacrylamide gels containing $0.1 \%$ SDS, and then transferred to polyvinylidene difluoride membranes (Beyotime Institute of Biotechnology). The membranes were then blocked with 5\% non-fat milk in Tris-buffered saline containing Tween-20 buffer (Shidai Biotechnology Co. Ltd, Shanghai, China). The blots were incubated with the primary antibodies diluted with blocking buffer (1:1,000 for p-JNK, JNK, p-Akt and Akt; $1: 300$ for $\mathrm{p} 53 ; 1: 200$ for $\mathrm{p}-\mathrm{ERK} 1 / 2$ and ERK1/2; 1:2,000 for $\beta$-actin; 1:1000 for caspase-3) at $4^{\circ} \mathrm{C}$ overnight, and then exposed to HRP-conjugated secondary antibodies $(1: 2,000)$ at room temperature for $2 \mathrm{~h}$ following washing with TBST 3 times. After washing with TBST for 3 times again, the bands on the membranes were subsequently visualized using enhanced chemiluminescence reagent (Beyotime Institute of Biotechnology), and images were captured using X-ray films. The pixel density of the proteins was quantified using ImageJ 1.47 software (National Institutes of Health, Bethesda, MD, USA).

Statistical analysis. Data are presented as the mean \pm standard deviation. Statistical significance of the data was determined using Student's t-test or one-way analysis of variance. Data analysis was conducted using GraphPad Prism 5 (Graphpad Software, Inc., La Jolla, CA, USA). P $<0.05$ was considered to indicate a statistically significant difference.

\section{Results}

Naringenin enhances curcumin-induced inhibition of THP-1 cell viability. To determine the role of naringenin in the AML cells, the THP-1 cells were treated with various concentrations of naringenin for $48 \mathrm{~h}$, and cell viability was determined. Naringenin reduced THP-1 cell viability in a dose-dependent manner, suggesting an inhibitory effect on cell growth (Fig. 1A). In addition, the cell viability of THP-1 cells treated with $100 \mu \mathrm{M}$ naringenin was evaluated at various time points; naringenin had a time-dependent effect on THP-1 cell viability (Fig. 1B). Since curcumin alone could inhibit AML cell viability, a lower dose of naringenin $(50 \mu \mathrm{M})$ was used to investigate the combination effects. A lower dose of curcumin $(20 \mu \mathrm{M})$ decreased cell viability by $\sim 15 \%$, and naringenin significantly enhanced the inhibitory effect induced by curcumin (Fig. 1C). Naringenin further decreased cell viability 
A

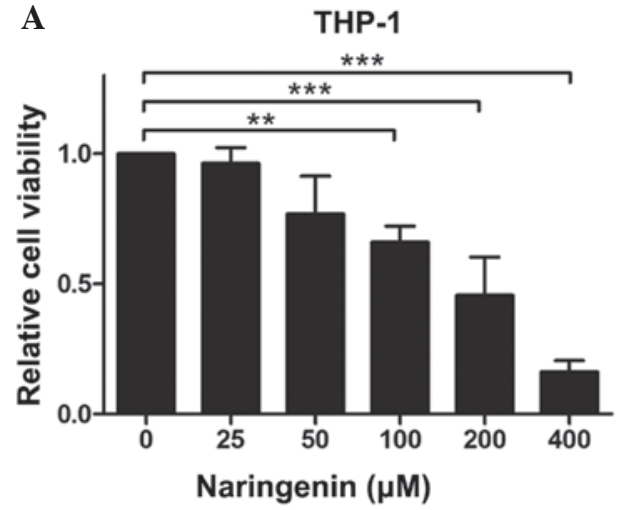

B

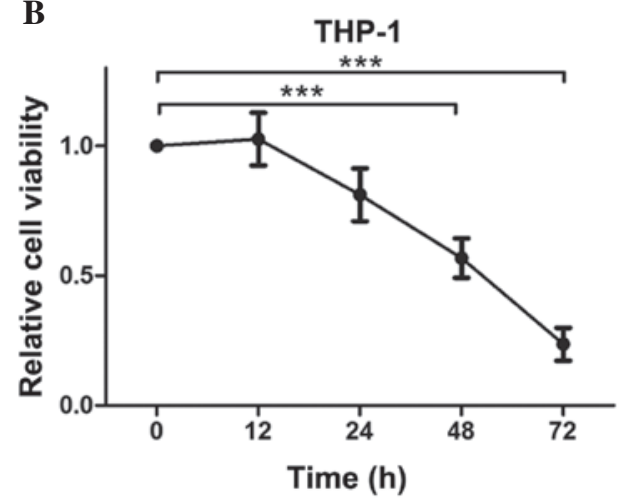

C

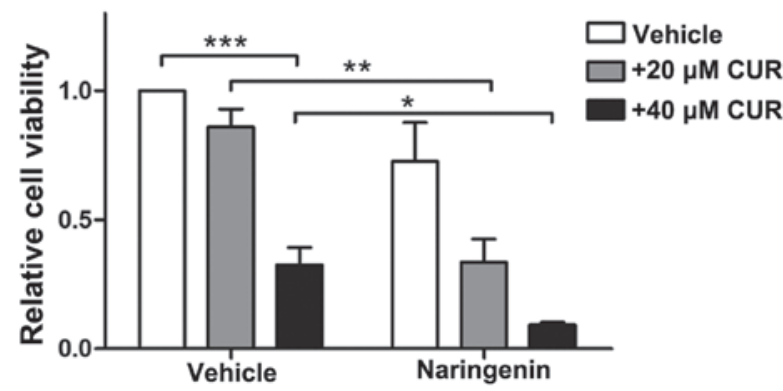

Figure 1. Naringenin could reduce THP-1 cell viability and enhance curcumin-induced inhibition of cell viability. (A) THP-1 cells were treated with naringenin at various concentrations for $48 \mathrm{~h}$ and cell viability was determined. (B) THP-1 cells were treated with $100 \mu \mathrm{M}$ naringenin for the indicated durations and cell viability was measured. (C) THP-1 cells were treated with or without $50 \mu \mathrm{M}$ naringenin and/or the indicated concentrations of curcumin (CUR) for $48 \mathrm{~h}$ and cell viability was evaluated. ${ }^{*} \mathrm{P}<0.05$, ${ }^{* * *} \mathrm{P}<0.01,{ }^{* * * *} \mathrm{P}<0.001$

when the cells were also treated with $40 \mu \mathrm{M}$ curcumin. These results indicate that naringenin may effectively enhance curcumin-induced inhibition of THP-1 cell viability.

Combined use of naringenin and curcumin induces $S$ and $G 2 / M$ phase cell cycle arrest. It has previously been reported that curcumin induces G1/S phase cell cycle arrest in mantle cell lymphoma (14), and naringenin induces G0/G1 phase cell cycle arrest in human epidermoid carcinoma cells (12). However, the effects of these two drugs on cell cycle distribution in THP-1 cells remains to be determined. The present study investigated the effects of various concentration of naringenin on cell cycle distribution in THP-1 cells. Naringenin induced cell cycle arrest
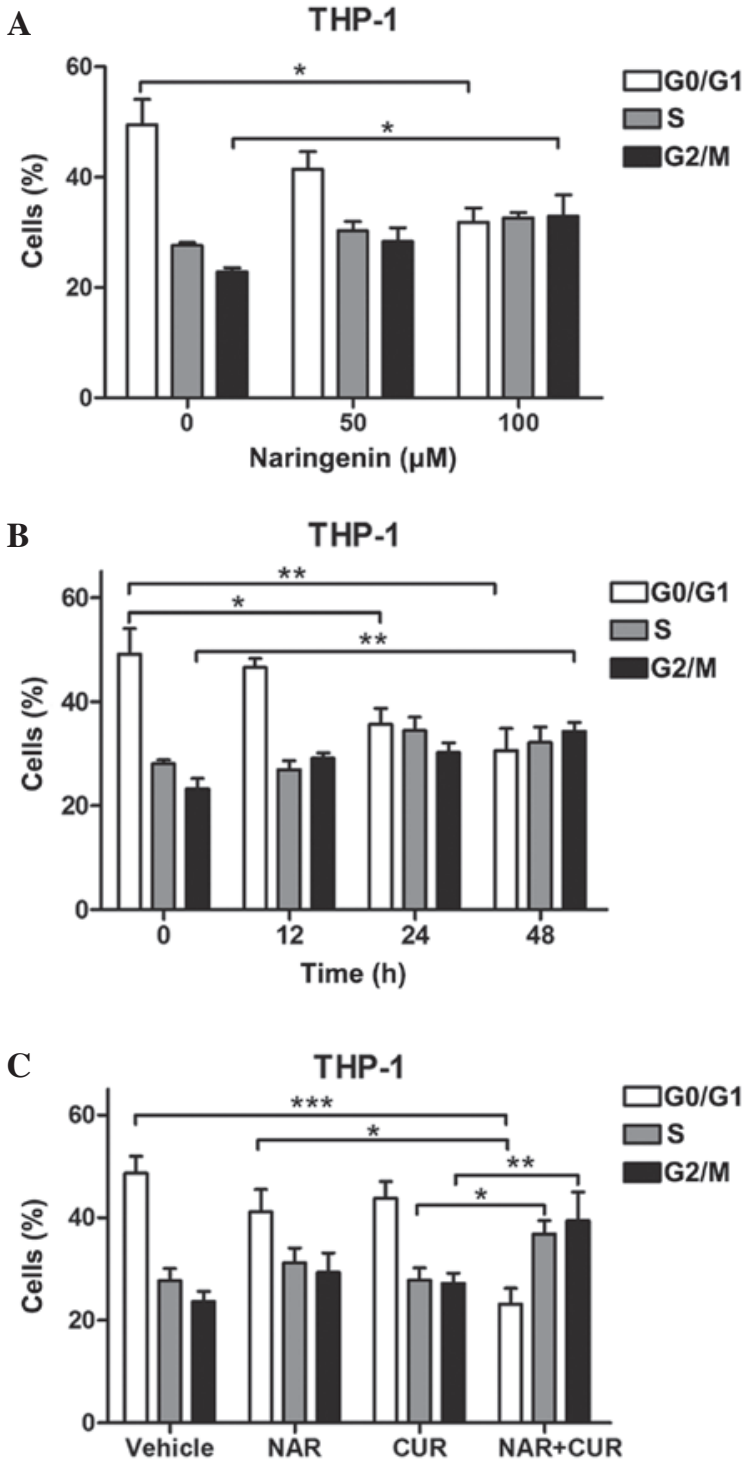

Figure 2. Naringenin (NAR) and curcumin (CUR) could induce $S$ phase and G2/M phase cell cycle arrest. (A) THP-1 cells were treated with 50 or $100 \mu \mathrm{M}$ NAR for $48 \mathrm{~h}$ and cell cycle distribution was determined. (B) THP-1 cells were treated with $100 \mu \mathrm{M}$ NAR for the indicated durations and cell cycle distribution was determined. (C) THP-1 cells were treated with or without $50 \mu \mathrm{M}$ NAR and/or $20 \mu \mathrm{M}$ CUR for $48 \mathrm{~h}$ and cell cycle distribution was determined. ${ }^{*} \mathrm{P}<0.05,{ }^{* *} \mathrm{P}<0.01,{ }^{* * * *} \mathrm{P}<0.001$.

at $\mathrm{G} 2 / \mathrm{M}$ phase, and the proportion of cells in $\mathrm{G} 0 / \mathrm{G} 1$ phase were significantly decreased following treatment with $100 \mu \mathrm{M}$ naringenin (Fig. 2A). However, these effects were not observed when the THP-1 cells were treated with a lower dose of naringenin (Fig. 2A). Cell cycle distribution was also determined at various time points after the cells were treated with $100 \mu \mathrm{M}$ naringenin; the number of cells in G0/G1 phase was significantly decreased after $24 \mathrm{~h}$ of treatment (Fig. 2B). However, cell cycle arrest at $\mathrm{G} 2 / \mathrm{M}$ phase was only detected after $48 \mathrm{~h}$ of treatment. No significant changes in cell cycle distribution were observed when the THP-1 cells were treated with naringenin $(50 \mu \mathrm{M})$ or curcumin $(20 \mu \mathrm{M})$ alone (Fig. 2C). However, the combination of these two drugs induced a significant increase of the number of cells in S phase and G2/M phase (Fig. 2C). These results indicate a synergistic effect of naringenin and curcumin on THP-1 cell cycle arrest at $\mathrm{S}$ phase and $\mathrm{G} 2 / \mathrm{M}$ phase. 

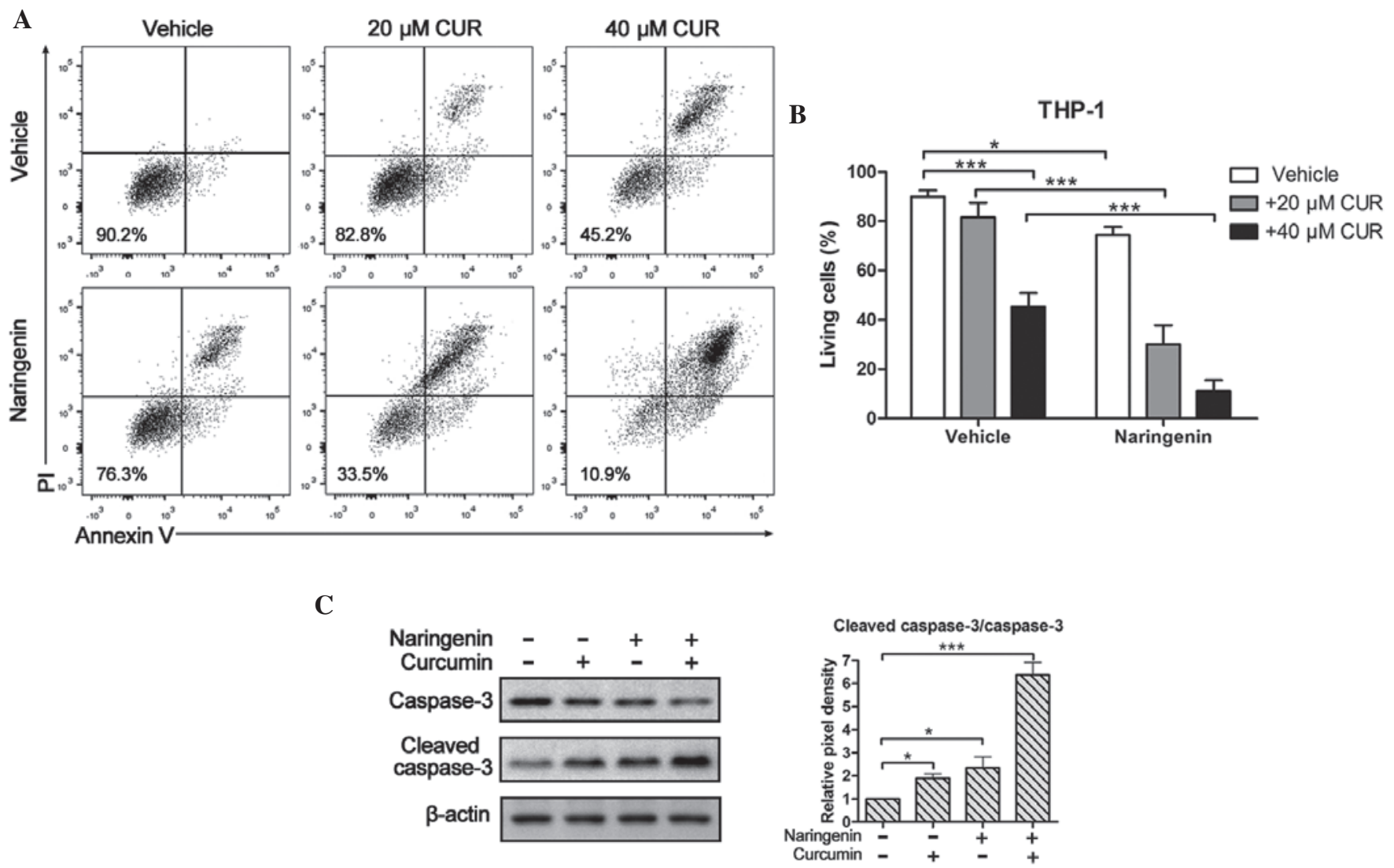

Figure 3. Naringenin and curcumin (CUR) induce apoptosis of THP-1 cells. (A) THP-1 cells were treated with or without $50 \mu \mathrm{M}$ naringenin and/or various concentrations of CUR for $48 \mathrm{~h}$ and cell apoptosis was measured using propidium iodide (PI)/Annexin-V staining. (B) The percentage of living cells following treatment with naringenin and CUR. (C) The expression levels of total and cleaved caspase-3 in THP-1 cells, as detected using western blotting following treatment with naringenin and CUR for $48 \mathrm{~h}$. The relative pixel densities are presented in a histogram. ${ }^{*} \mathrm{P}<0.05,{ }^{* * *} \mathrm{P}<0.01,{ }^{* * * *} \mathrm{P}<0.001$.

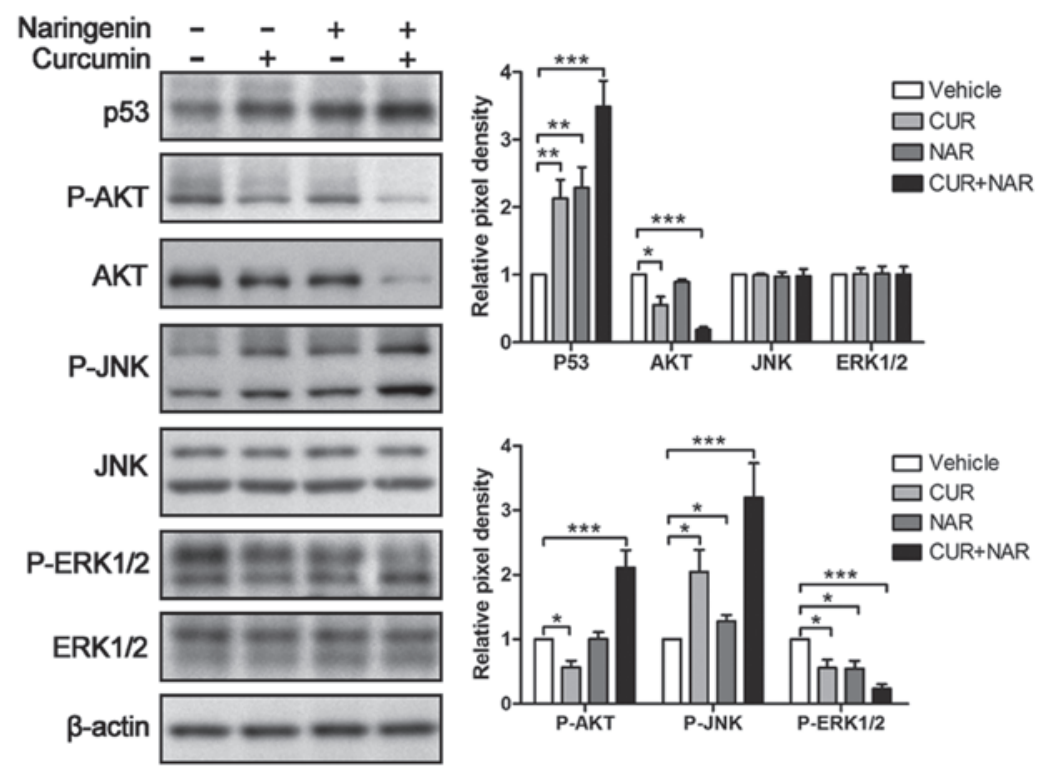

Figure 4. Naringenin (NAR) and curcumin (CUR) induce changes in p53, Akt, c-Jun N-terminal kinases (JNK) and extracellular signal-regulated kinases (ERK) $1 / 2$ expression. THP-1 cells were treated with or without $50 \mu \mathrm{MNAR}$ and/or $20 \mu \mathrm{M}$ CUR for $24 \mathrm{~h}$, and the expression of p53, Akt, JNK and ERK1/2, and the phosphorylation (p) of Akt, JNK and ERK1/2 were detected by western blotting. Relative pixel densities are presented in histograms. Protein expression was normalized to $\beta$-actin and the phosphorylated proteins were normalized to their total proteins. ${ }^{*} \mathrm{P}<0.05,{ }^{* * *} \mathrm{P}<0.01,{ }^{* * * *} \mathrm{P}<0.001$.

Naringenin and curcumin induce THP-1 cell apoptosis. Since the combined use of naringenin and curcumin was able to induce cell viability inhibition, the effect of these two drugs on apoptosis was also investigated using PI/Annexin-V staining. Treatment with curcumin $(20$ or $40 \mu \mathrm{M})$ and naringenin $(50 \mu \mathrm{M})$ resulted in a significant increase in the number of 
apoptotic cells, whereas no significant increase was detected when the cells were treated with curcumin alone at a lower concentration $(20 \mu \mathrm{M})$ (Fig. 3A and B). However, treatment with naringenin increased the percentage of apoptotic cells by $\sim 50 \%$, as compared with that induced by a lower dose of curcumin (Fig. 3A and B). In addition, the expression levels of cleaved caspase- 3 were increased in the THP-1 cells following treatment with naringenin and curcumin (Fig. 3C), as compared with in the cells treated with naringenin or curcumin alone. These results suggest that treatment with curcumin and naringenin may enhance apoptosis.

Naringenin and curcumin inhibit Akt and ERK1/2, and promote $J N K$ and $p 53$. The present study confirmed that naringenin was able to enhance curcumin-induced apoptosis; therefore, the expression levels of proteins involved in pathways associated with apoptosis, including p53, Akt, JNK and ERK1/2 were determined. Treatment with curcumin or naringenin alone increased p53 expression; however, the expression levels of p53 were markedly increased when the two drugs were used. The expression levels of p-Akt and total Akt were markedly reduced following combined treatment with curcumin and naringenin, whereas the ratio of p-Akt/Akt was increased. Treatment with curcumin alone reduced p-Akt and total Akt expression levels, and naringenin alone did not effect on them.

Treatment with curcumin or naringenin alone marginally increased the phosphorylation of JNK, whereas the highest expression levels of $\mathrm{p}$-JNK were detected when the THP-1 cells were treated with a combination of curcumin and naringenin. The expression levels of $\mathrm{p}$-ERK1/2 were reduced following treatment with curcumin or naringenin alone, and co-treatment enhanced the inhibition. The expression levels of total JNK and ERK1/2 were not altered following any of the three treatment conditions. These results indicate that naringenin and curcumin may inhibit the activation of the Akt and ERK pathways, and promote the expression of p53 and activation of the JNK pathway, leading to cell apoptosis.

\section{Discussion}

The present study investigated the combination effects of curcumin and naringenin on cell viability, cell cycle distribution and apoptosis, and on the activation of numerous pathways in the THP-1 human AML cell line. The results demonstrated that naringenin was able to enhance curcumin-induced apoptosis by altering the activation of Akt, ERK, JNK and p53 pathways. The present study revealed the promising anticancer effects of these two agents in THP-1 cells.

Curcumin is a well-investigated drug, which exerts anticancer effects in various types of cancer, including lung cancer $(15,16)$, gastric carcinoma (17), renal cell carcinoma (18), ovarian cancer (19), liver cancer $(20,21)$, breast cancer (22), AML (23) and acute lymphoblastic leukemia (24). It has previously been reported that curcumin may induce cell cycle arrest at $\mathrm{S}$ phase and $\mathrm{G} 2 / \mathrm{M}$ phase in breast cancer cells (25), or at G1/S phase in mantle cell lymphoma (14). However, the results of the present study did not detect cell cycle arrest when the THP-1 cells were treated with curcumin alone, and this may be due to the low dose of curcumin $(20 \mu \mathrm{M})$ used in the present study. Curcumin has been shown to affect the JNK, Akt, AMP-activated protein kinase, ERK, p53, nuclear factor- $\kappa \mathrm{B}$ and signal transducer and activator of transcription 3 pathways to modulate growth of tumor cells (8). The present study demonstrated that curcumin was able to increase the expression levels of p53 and the activation of JNK, and could reduce Akt expression and ERK1/2 activation in THP-1 cells.

The anticancer effects of naringenin have been investigated in numerous types of solid cancer, and the results have demonstrated that it may induce tumor growth inhibition and apoptosis of tumor cells $(3,13)$. However, the anticancer effects of naringenin in AML cells have not been fully determined. The results of the present study demonstrated that naringenin was able to inhibit cell growth and induce apoptosis in the THP-1 AML cell line. A previous study reported that naringenin downregulates Akt activation and induces THP-1 cell apoptosis (26). In the present study, naringenin was shown to inhibit Akt expression and ERK1/2 activation in THP-1 cells following treatment with curcumin.

Since naringenin and curcumin exert anticancer effects, the present study also examined the combined effects in THP-1 cells. Co-treatment with naringenin and curcumin induced an increased rate of cell apoptosis and further inhibited the viability of THP-1 cells. Treatment with low doses of naringenin or curcumin did not induce cell cycle arrest, whereas co-treatment with naringenin and curcumin induced cell cycle arrest at $\mathrm{S}$ phase and $\mathrm{G} 2 / \mathrm{M}$ phase, thus indicating the presence of a combination effect. Furthermore, naringenin and curcumin co-treatment decreased the activation of ERK1/2, and increased JNK activation and p53 expression. Activation of ERK and Akt pathways may induce cell survival; however, in the present study, inhibition of these two pathways was observed, suggesting that naringenin and curcumin may induce apoptosis through inhibition of ERK and Akt. Furthermore, p53 expression and JNK activation can initiate apoptotic pathways, and naringenin and curcumin were shown to induce these two pathways, which may result in cell apoptosis.

In conclusion, co-treatment with naringenin and curcumin has been demonstrated to exert anticancer effects in THP-1 cells, by inducing cell apoptosis through numerous pathways. Inhibition of Akt and ERK pathways, and promotion of p53 and JNK pathways may be involved in naringenin and curcumin-induced cell apoptosis in AML cells. The results of the present study warrant further investigation to confirm the anti-cancer effects of this treatment combination in vivo.

\section{Acknowledgements}

The authors of the present study would like to thank all laboratory staff for the critical reading of the manuscript. The present study was supported by the Guangdong Provincial Foundation for key specialty Hematology and Science \& Technology Project of Guangdong Province Social Development Foundation (grant no. 2013B021800103).

\section{References}

1. Steffen B, Müller-Tidow C, Schwäble J, Berdel WE and Serve H: The molecular pathogenesis of acute myeloid leukemia. Crit Rev Oncol Hematol 56: 195-221, 2005. 
2. Deschler B and Lübbert M: Acute myeloid leukemia: Epidemiology and etiology. Cancer 107: 2099-2107, 2006.

3. Zhou H, Beevers CS and Huang S: The targets of curcumin. Curr Drug Targets 12: 332-347, 2011.

4. Aggarwal BB and Sung B: Pharmacological basis for the role of curcumin in chronic diseases: An age-old spice with modern targets. Trends Pharmacol Sci 30: 85-94, 2009.

5. Sharma RA, Steward WP and Gescher AJ: Pharmacokinetics and pharmacodynamics of curcumin. Adv Exp Med Biol 595: 453-470, 2007.

6. Gandhy SU, Kim K, Larsen L, Rosengren RJ and Safe S: Curcumin and synthetic analogs induce reactive oxygen species and decreases specificity protein $(\mathrm{Sp})$ transcription factors by targeting microRNAs. BMC Cancer 12: 564, 2012.

7. Liao YF, Hung HC, Hour TC, Hsu PC, Kao MC, Tsay GJ and Liu GY: Curcumin induces apoptosis through an ornithine decarboxylase-dependent pathway in human promyelocytic leukemia HL-60 cells. Life Sci 82: 367-375, 2008.

8. Ravindran J, Prasad S and Aggarwal BB: Curcumin and cancer cells: How many ways can curry kill tumor cells selectively? AAPS J 11: 495-510, 2009.

9. Sabarinathan D, Mahalakshmi P and Vanisree AJ: Naringenin, a flavanone inhibits the proliferation of cerebrally implanted C6 glioma cells in rats. Chem Biol Interact 189: 26-36, 2011.

10. Ekambaram G, Rajendran P, Magesh V and Sakthisekaran D: Naringenin reduces tumor size and weight lost in $\mathrm{N}$-methyl-N'-nitro-N-nitrosoguanidine-induced gastric carcinogenesis in rats. Nutr Res 28: 106-112, 2008.

11. Qin L, Jin L, Lu L, Lu X, Zhang C, Zhang F and Liang W: Naringenin reduces lung metastasis in a breast cancer resection model. Protein Cell 2: 507-516, 2011.

12. Ahamad MS, Siddiqui S, Jafri A, Ahmad S, Afzal M and Arshad M: Induction of apoptosis and antiproliferative activity of naringenin in human epidermoid carcinoma cell through ROS generation and cell cycle arrest. PLoS One 9: e110003, 2014.

13. Arul D and Subramanian P: Naringenin (citrus flavonone) induces growth inhibition, cell cycle arrest and apoptosis in human hepatocellular carcinoma cells. Pathol Oncol Res 19: 763-770, 2013

14. Shishodia S, Amin HM, Lai R and Aggarwal BB: Curcumin (diferuloylmethane) inhibits constitutive NF-kappaB activation, induces G1/S arrest, suppresses proliferation, and induces apoptosis in mantle cell lymphoma. Biochem Pharmacol 70: $700-713,2005$
15. Li Y, Zhang S, Geng JX and Hu XY: Curcumin inhibits human non-small cell lung cancer A549 cell proliferation through regulation of $\mathrm{Bcl}-2 / \mathrm{Bax}$ and cytochrome $C$. Asian Pac J Cancer Prev 14: 4599-4602, 2013

16. Mehta HJ, Patel V and Sadikot RT: Curcumin and lung cancer - a review. Target Oncol 9: 295-310, 2014.

17. Cao A, Li Q, Yin P, Dong Y, Shi H, Wang L, Ji G, Xie J and Wu D: Curcumin induces apoptosis in human gastric carcinoma AGS cells and colon carcinoma HT-29 cells through mitochondrial dysfunction and endoplasmic reticulum stress. Apoptosis 18: 1391-1402, 2013.

18. Bill MA, Nicholas C, Mace TA, Etter JP, Li C, Schwartz EB, Fuchs JR, Young GS, Lin L, Lin J, et al: Structurally modified curcumin analogs inhibit STAT3 phosphorylation and promote apoptosis of human renal cell carcinoma and melanoma cell lines. PLoS One 7: e40724, 2012.

19. Cai YY, Lin WP, Li AP and Xu JY: Combined effects of curcumin and triptolide on an ovarian cancer cell line. Asian Pac J Cancer Prev 14: 4267-4271, 2013

20. Dai XZ, Yin HT, Sun LF, Hu X, Zhou C, Zhou Y, Zhang W, Huang XE and Li XC: Potential therapeutic efficacy of curcumin in liver cancer. Asian Pac J Cancer Prev 14: 3855-3859, 2013.

21. Du Q, Hu B, An HM, Shen KP, Xu L, Deng S and Wei MM: Synergistic anticancer effects of curcumin and resveratrol in Hepa1-6 hepatocellular carcinoma cells. Oncol Rep 29: 1851-1858, 2013.

22. Mo N, Li ZQ, Li J and Cao YD: Curcumin inhibits TGF- $\beta 1$-induced MMP-9 and invasion through ERK and Smad signaling in breast cancer MDA-MB-231 cells. Asian Pac J Cancer Prev 13: 5709-5714, 2012.

23. Yang CW, Chang CL, Lee HC, Chi CW, Pan JP and Yang WC Curcumin induces the apoptosis of human monocytic leukemia THP-1 cells via the activation of JNK/ERK pathways. BMC Complement Altern Med 12: 22, 2012.

24. Wang H, Geng QR, Wang L and Lu Y: Curcumin potentiates antitumor activity of L-asparaginase via inhibition of the AKT signaling pathway in acute lymphoblastic leukemia. Leuk Lymphoma 53: 1376-1382, 2012.

25. Ke CS, Liu HS, Yen CH, Huang GC, Cheng HC, Huang CY and Su CL: Curcumin-induced Aurora-A suppression not only causes mitotic defect and cell cycle arrest but also alters chemosensitivity to anticancer drugs. J Nutr Biochem 25: 526-539, 2014.

26. Park JH, Jin CY, Lee BK, Kim GY, Choi YH and Jeong YK: Naringenin induces apoptosis through downregulation of Akt and caspase-3 activation in human leukemia THP-1 cells. Food Chem Toxicol 46: 3684-3690, 2008 